\title{
VIRTUAL TESTING OF LARGE COMPOSITE STRUCTURES: A MULTIPLE LENGTH/TIME-SCALE FRAMEWORK
}

\author{
LUIGI GIGLIOTTI \\ Department of Aeronautics, Imperial College London, South Kensington Campus \\ London, SW7 2AZ, United Kingdom \\ SILVESTRE T. PINHO \\ Department of Aeronautics, Imperial College London, South Kensington Campus \\ London, SWr 2AZ, United Kingdom
}

\begin{abstract}
This paper illustrates a multiple length/time-scale framework for the virtual testing of large composite structures. Such framework hinges upon a Mesh Superposition Technique (MST) for the coupling between areas of the structure modelled at different length-scales and upon an efficient solid-to-shell numerical homogenization which exploits the internal symmetries of Unit Cells (UCs). Using this framework, it is possible to minimize the areas of the structure modelled at the lowest- (and computationally demanding) scales and the computational cost required to calculate the homogenised to be used in the higher-scales subdomains of multiscale FE models, as well as to simulate the mechanical response of different parts of the structure using different solvers, depending on where they are expected to provide the most computationally efficient solution. The relevance and key-aspects of the multiple length/time-scale framework are demonstrated through the analysis of a real-sized aeronautical composite component.
\end{abstract}

Keywords: Virtual Testing; Large composite structures; Multiple length/time-scale.

\section{Introduction}

\subsection{Virtual testing of large composite structures}

To address complex industrial structural challenges, such as the design of the composite central wing box of the Airbus $\mathrm{A} 380^{1}$, virtual testing methods have been exploited $^{2}$. The extensive use of virtual testing based on nonlinear FE analyses is envisioned to be a key-aspect towards an increased confidence in the real-scale and expensive structural tests required for certification; furthermore, virtual testing provides useful insight into the likelihood, causes and consequences of structural failure $^{3,4,5}$. However, to be fully established in structural design and certification, virtual testing methods need to be validated against all level of structural testing, from the coupon-level (e.g. material specimens) to the system-level (e.g. wing or fuselage $)^{6}$.

Within this framework, the virtual testing of large-scale composite structures for industrial applications entails significant challenges; the latter are primarily ascribable to the inherently multiscale nature of composite materials and, as a 
result, to their highly complex failure modes ${ }^{7}$. For the efficient structural design of large composite components, their virtual testing often requires that different parts of the structure are modelled at multiple length- and time-scales, eventually even using different physics. Therefore, it is crucial to develop:

(i) suitable techniques for coupling areas of the structure modelled at different length-scales, i.e. discretized using different finite element types;

(ii) numerical methods to efficiently compute equivalent homogenized properties to be used in both 2D FE models and in the lower-scale subdomains of multiscale FE models of large composite components.

\subsection{Multiscale coupling}

The coupling of subdomains discretized with finite elements of different physical dimension/formulation can introduce artificial stresses at the shared boundaries ${ }^{8,9}$. Hence, the stress and strain fields within the structure and its mechanical response may not be correctly simulated. This may lead, in problems involving failure, to a low-fidelity damage pattern prediction: furthermore, in dynamic problems, the interfaces between differently-discretized subdomains might artificially reflect stress waves ${ }^{11,12}$.

Global/local approaches can be categorized as coupled and uncoupled approaches ${ }^{10}$. In the former, the coupling between the local and global models (discretized using different finite elements) is enforced through Multi-Point Constraints (MPC). Numerous mixed-dimensional coupling techniques based on MPCs are available in literature ${ }^{8,13,14}$. Compared to standard solid-to-shell coupling, these methodologies are shown to attenuate the undesirable stress disturbances at the interface between differently-discretized subdomains; nevertheless, the derivation of the suitable MPC equations for generally orthotropic materials may become computationally impracticable ${ }^{14}$. Alternatively, Dávila ${ }^{9}$ demonstrated that the use of ad-hoc transition elements allows to faithfully resolve the stress and strain fields within both the local and global models in the proximity of the interfaces.

In uncoupled global/local approaches, the displacements/tractions obtained from a higher-scale global model are used to prescribe the boundary conditions for the lower-scale local model. The series of higher-scale analysis followed by a lower scale analysis can be run once (a typical example is the Submodelling technique available in Abaqus ${ }^{15}$ ), or iteratively until convergence of the forces and moments on the local/global interface is obtained. As a result, these approaches are difficult to automate and pose significant challenges in terms of computational resources; furthermore, they might be inaccurate if damage and failure propagation within the local model significantly affect the response of the global model. Reinoso et al. ${ }^{16,17}$ compared the Submodelling technique (uncoupled approach) to Shell-to-Solid coupling (coupled approach). In the latter works, stress disturbances were observed at the local/global boundaries for both approaches; as a result, the correct identification of the damage extension might be jeopardized. 
To enhance the computational efficiency of multi-scale approaches, it is desirable that the size of the lower-scale model is kept to a minimum. However, the global/local transition should be sufficiently distant from any perturbation such as boundaries or damaged zones which could potentially interact with the stress disturbance at the discretization transition. The influence of the distance of the global/local transition from the delamination front was investigated by Krueger et al. for the cases of delaminated test specimens ${ }^{18,19}$ as well as skin/stringer debonding in an composite aircraft component ${ }^{20,21}$.

The need of having the global/local transition at a sufficient distance from any boundaries/damaged areas often leads to overly large models at the lower-scales; as a result, the computational efficiency of the analysis might not be optimal. Thus, to fully exploit the computational advantages provided by multiscale approaches, it is of paramount importance to use a local/global coupling technique which:

(i) avoids the presence of artificial stress disturbances, as well as the unrealistic stress wave reflections observed at the boundaries between differently discretized subdomains;

(ii) allows to minimize the size of the areas that are required to be modelled at the lower length-scales.

\subsection{Solid-to-shell homogenization}

For the virtual testing of large composite structures, FE (preferably 2D) models with homogenised material properties are usually preferred to high-fidelity 3D FE models. Furthermore, shell-based FE models with equivalent homogenized properties are used in the higher-scale subdomains of multiscale FE models of large composite components.

The numerical homogenization of periodic structures is often carried out through the analysis of the mechanical response of Unit Cell (UC) models, where the composite microstructure is modelled at the lowest length/scale of interest. Numerous studies focused on the determination and use of $\mathrm{UCs}^{22,23}$, as well as on the appropriate periodic boundary conditions (PBCs) that need to be applied for their analysis ${ }^{24,25,26,27}$. However, as a result of the increasing complexity of composite microstructures, e.g. textile and NCF composites, pin-reinforced sandwich structures, etc., the computational cost associated to modelling/meshing and analysis might become burdensome.

To circumvent this difficulty, several authors have proposed to exploit (whenever they exist) internal symmetries of the UCs, thus enabling a significant reduction of the analysis domain. Analysis domains smaller than UCs are denoted as reduced Unit Cells (rUCs) and have successfully been used for the analysis UD composites $^{28}$, particle-reinforced composites ${ }^{29}$ and textile composites ${ }^{32,31,30}$. However, in these works address the issue of using rUCs for obtaining equivalent 3D homogenised properties, without considering the computation of equivalent 2D homogenised properties. 
The efficient computation of the equivalent homogenized properties to be used in both 2D FE models and in the higher-scale subdomains of multiscale FE models of large composite components hinges upon the development of a mathematical framework which:

(i) enables the direct two-scale (solid-to-shell) homogenization of periodic structures, including their bending and twisting response;

(ii) enables the use of rUCs.

\subsection{Structure of this paper}

In this paper, we illustrate the capabilities of a multiple length/time-scale framework for the virtual testing of large composite structures.

The multiple length/time-scale framework is described in Section 2 and its primitives are illustrated and discussed, respectively in Section 3 and Section 4, through the simulation of a real-sized helicopter rotor blade subjected to a low-velocity impact; conclusions are drawn in Section 5.

\section{Multiple length/time scale framework}

The multiple length/time-scale framework used in this paper, and graphically illustrated in Fig. 1, consists of (i) a Mesh Superposition Technique (MST) ${ }^{33}$ for the progressive transition between subdomains discretized with finite elements of different physical dimension/formulation and (ii) a mathematical framework which exploit UCs internal symmetries in the context of the direct solid-to-shell homogenization of periodic structures ${ }^{34}$.

The key-aspects of the MST are:

(i) unlike conventional solid-to-shell coupling techniques based on a sudden discretization-transition, the MST eliminates the artificial stress disturbances at the shared boundaries between differently discretized subdomains;

(ii) using the MST, the size of the lower-scale models (the most computationally demanding) can be minimized without jeopardizing the response at the global/local transition. Therefore, compared to conventional solid-to-shell coupling techniques based on a sudden discretization-transition the MST enables a high-fidelity damage pattern prediction at a lower computational cost;

(iii) the progressive transition provided by the MST mitigates the spurious reflections of stress waves at the interfaces between differently-discretized subdomains (see example in Appendix A);

(iv) the MST can be used in combination with an implicit/explicit co-simulation technique $^{35,36}$, for a multiple time/length-scale analysis. The capability provided by the MST to minimize the size of the lower-scale models allows to 

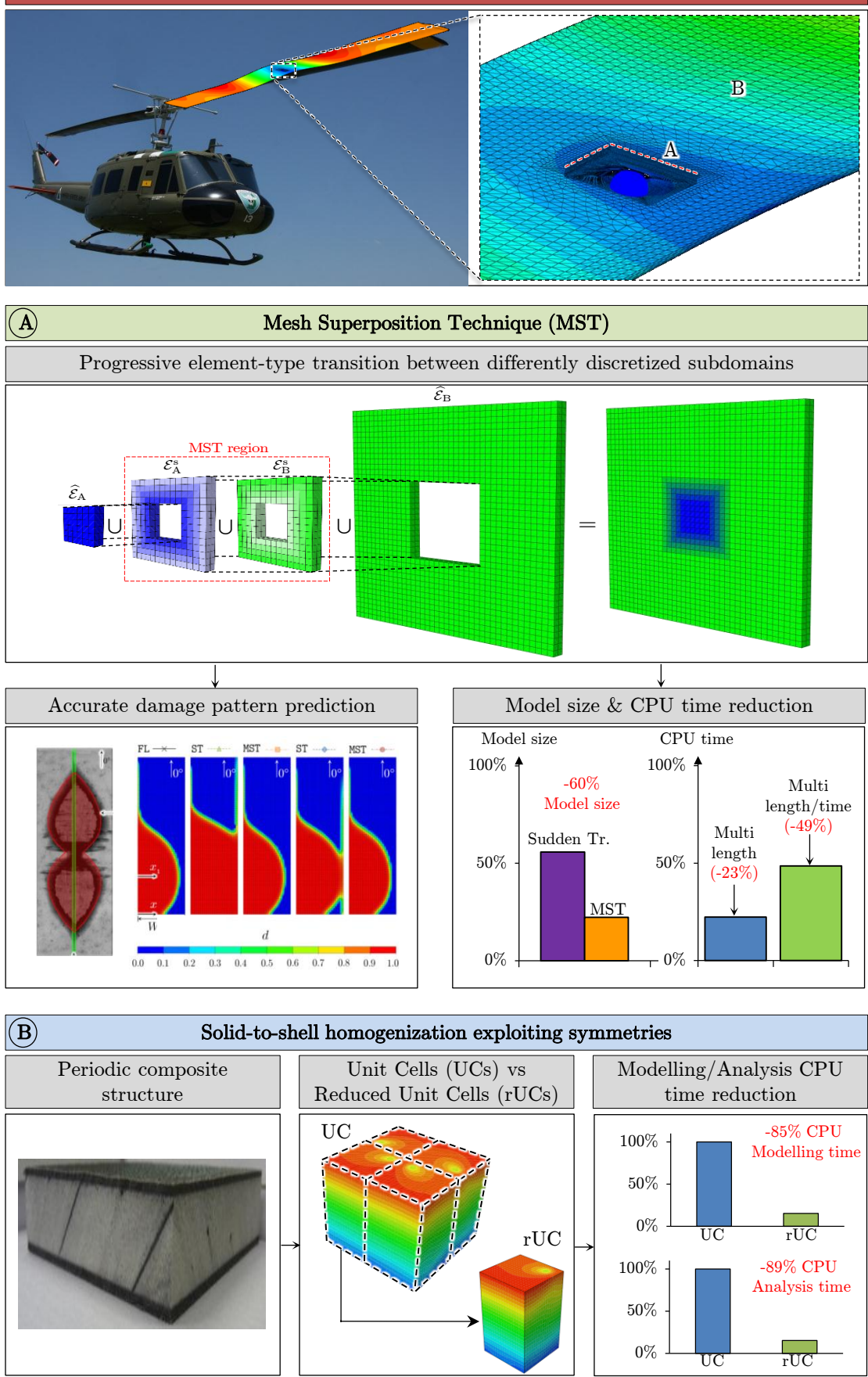

Fig. 1. Multiple length/time-scales framework for the virtual testing of large composite components. Such framework consists of a Mesh Superposition Technique for coupling differentlydiscretized subdomains (A) and on the exploitation of symmetries in the solid-to-shell numerical homogenization of periodic structures. 
maximize the computational efficiency of the implicit/explicit co-simulation technique; hence, in the context of a multiple length/time scale analysis, the use of the MST for the length-scale transitions (instead of a sudden discretization-transition) results into a significant computational cost reduction.

Regarding the exploitation of symmetries in the solid-to-shell homogenization of periodic structure, the following points should be highlighted:

(i) the framework proposed in Ref. 34 leads to the derivation of the exact periodic boundary conditions that apply to rUCs and enable the numerical solid-to-shell homogenization of periodic structures, including their bending and twisting response.

(ii) no limitations on the deformed/undeformed shape of the rUCs, as well as to the nonlinear behaviour at the lowest length-scale were made (provided there is no localization ${ }^{37}$ );

(iii) when comparing results obtained using conventional UCs with those obtained with rUCs, in the latter case time savings of about $90 \%$ can be achieved in the analysis CPU time, as well as in the modelling/meshing CPU time.

\section{Multiple length/time-scale simulation of a large aeronautical component}

\subsection{Problem description}

The multiple length/time-scale framework detailed in Section 2 is applied to the analysis of a low-velocity impact on real-sized helicopter rotor blade. The latter is idealized as an hollow structure with dimensions provided in Fig. 2. The profile of the rotor blade corresponds to the NASA/Langley Whitcomb integral supercritical airfoil $^{38}$. The rotor blade is impacted with a $2.5 \mathrm{~kg}$ impactor of diameter equal to $40 \mathrm{~mm}$ and a $12 \mathrm{~J}$ impact energy was considered.

The profile of the rotor blade is assumed to be made of a composite sandwich structure $^{39,40}$ with pin-reinforced foam core ${ }^{41}$; relevant geometrical and material properties of the pin-reinforced sandwich structure can be found in Ref. 34. The material considered for the pin-reinforcements is the carbon-epoxy T300/913 ${ }^{42}$. 


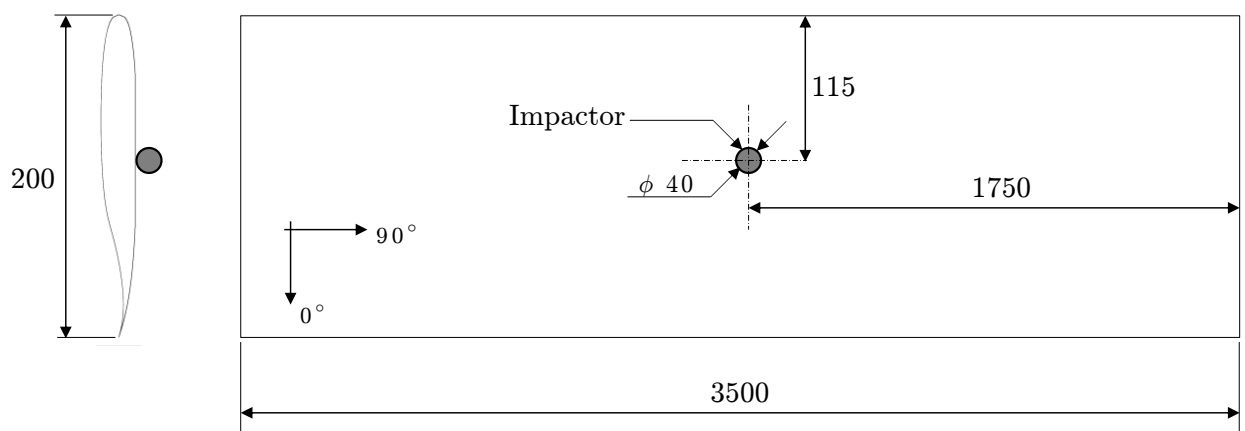

Fig. 2. Schematic (not in scale) of the helicopter rotor blade considered in this study; dimensions are in $\mathrm{mm}$.

\subsection{Multi length/time-scale FE model}

\subsection{Implicit \& Explicit FE submodels}

The multiple time-scale connotation of the framework described in Section 2 consists in simulating the mechanical response of different portions of the structure using different solvers, depending on where they are expected to provide the most computationally efficient solution. Generally, complex material failure, contact interactions and highly nonlinear response are best analysed using FE solvers based on explicit time-integration schemes, e.g. Abaqus/Explicit, while the elastic behaviour of light and stiff components can, more efficiently, be simulated with FE solvers using implicit time-integration schemes, e.g. Abaqus/Standard.

Using different $\mathrm{FE}$ solvers implies the definition of multiple time-scales at which the structural response is analysed: (i) an explicit/micro time-scale, characterized by a high number of short and relatively inexpensive time-steps and (ii) an implicit/macro time-scale, characterized by a reduced number of larger time-steps, due to the unconditional stability of implicit solvers.

Therefore, for the problem described in Section 3.1, the FE model of the rotor blade (see Fig. 3) consists of two separate FE submodels:

(i) an explicit submodel for the area of the rotor blade surrounding the impact location (where contact interactions and complex failure modes are expected); within this submodel, different parts of the structure are modelled at different length-scales as described in Section 3.4;

(ii) an implicit submodel for the remaining of the structure, whose deformation remains elastic throughout the entire analysis.

In the implicit $\mathrm{FE}$ subdomain, the rotor blade is discretized using first-order triangular shell elements (S3). The equivalent homogenised 2D behaviour, i.e. the entire ABD matrix of the pin-reinforced sandwich structure is defined exploiting 


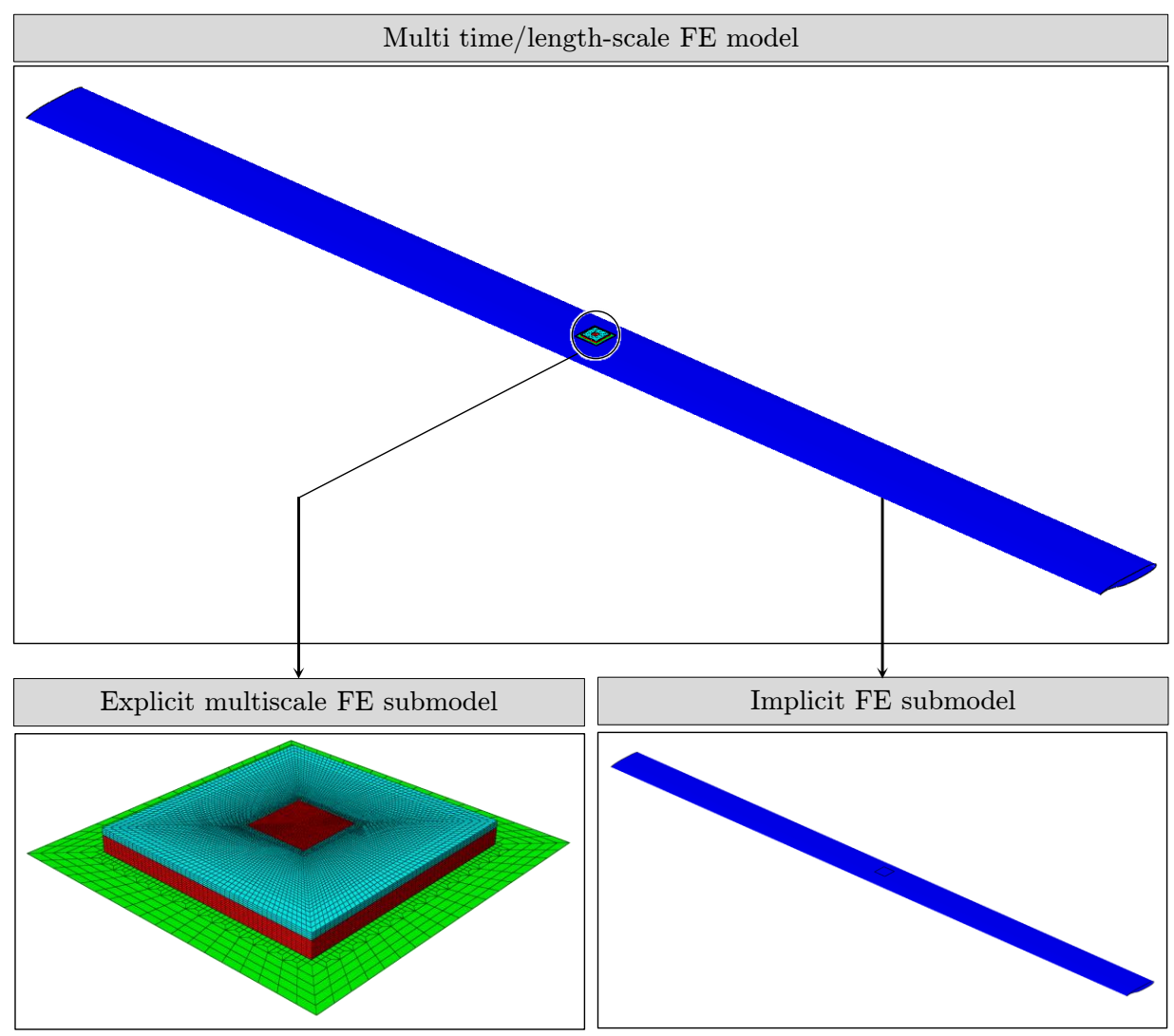

Fig. 3. Implicit/explicit submodels of a multiple length/time-scale FE model of an helicopter rotor blade. The area surrounding the impact location is analysed using an explicit solver, to exploit its capabilities to better handle contact interactions and complex failures modes; the response of the remaining of the structure (the largest part) is more efficiently simulated using an implicit solver.

the solid-to-shell homogenization described in Section 2. The meshing of the entire rotor blade required $63744 \mathrm{~S} 3$ elements.

In this example, the multiple length/time-scale analysis is performed exploiting Abaqus built-in Co-Simulation Engine $(\mathrm{CSE})^{15}$; the simulation of the mechanical response of the implicit and explicit submodels is performed using, respectively, Abaqus/Explicit and Abaqus/Standard.

\subsection{Multiscale explicit FE submodel}

Within the explicit submodel, different parts of the structure are modelled at different length-scales and the coupling between them is performed using the $\mathrm{MST}^{33}$. The size of the differently-discretized subdomains needs to be established a priori, i.e. before the analysis, for example by exploiting analytical methods to estimate 
the expected size of the damaged areas ${ }^{50}$.

For the example described in the present paper, we considered three different regions where the structure is modelled at different length-scales (see Fig. 4):

\section{Meso-scale subdomain}

Within this subdomain, the structure is modelled at the lowest lengthscale. The plies in the composite facesheets, the interfaces between them and between the facesheets and the core, as well as the pin-reinforcements within the core, are modelled individually.

Eight-noded reduced-integration solid elements (C3D8R) with enhanced hourglass control were used for the discretization of the composite plies, pinreinforcements and core layer: eight-noded cohesive elements (COH3D8) were used to account for possible delamination (between composite plies) and debonding (between the facesheets and the core). To simplify the meshing of the pin-reinforced core, the pins' finite elements were embedded within the foam core exploiting the Embedded Element Method (EEM) available in Abaqus. This method has been already used for modelling pinreinforced cores ${ }^{44}$ and textile composites ${ }^{45}$. The discretization of the mesoscale subdomain (including the MST transition regions) required 71896 C3D8R elements and 11520 COH3D8 elements.

The complex shape of the pin-reinforcements (i.e. misalignments, bended ends, etc.) is also explicitly modelled. Within the meso-scale subdomain, several failure modes are accounted for (see Fig. 4), i.e

(i) delamination between composite plies, as well as debonding between the composite facesheets and the core, using multiple layers of cohesive elements with a mixed-mode bilinear traction-separation law to simulate the softening and fracture response ${ }^{46,47}$;

(ii) crushing of the foam core ${ }^{48}$;

(iii) failure of the pin reinforcements ${ }^{49}$;

\section{Meso/Macro scale region}

Within this subdomain, the composites facesheets, the core, the interface between them, as well as the pin-reinforcements within the core layer, are explicitly modelled.

Eight-noded reduced-integration continuum shell elements (SC8R) with enhanced hourglass control were used for the discretization of the composite facesheets while eight-noded reduced-integration solid elements (C3D8R) were used for the discretization of the pin-reinforcements and of the core. As in the meso-scale subdomain, the pins' finite elements were embedded within the core exploiting the EEM. The discretization of the meso-scale subdomain (including the MST transition regions) required 181952 C3D8R elements, 54784 SC8R elements and 11520 COH3D8 elements.

Within the meso/macro-scale subdomain, the only failure model accounted for is the debonding between the core and the composite facesheets 


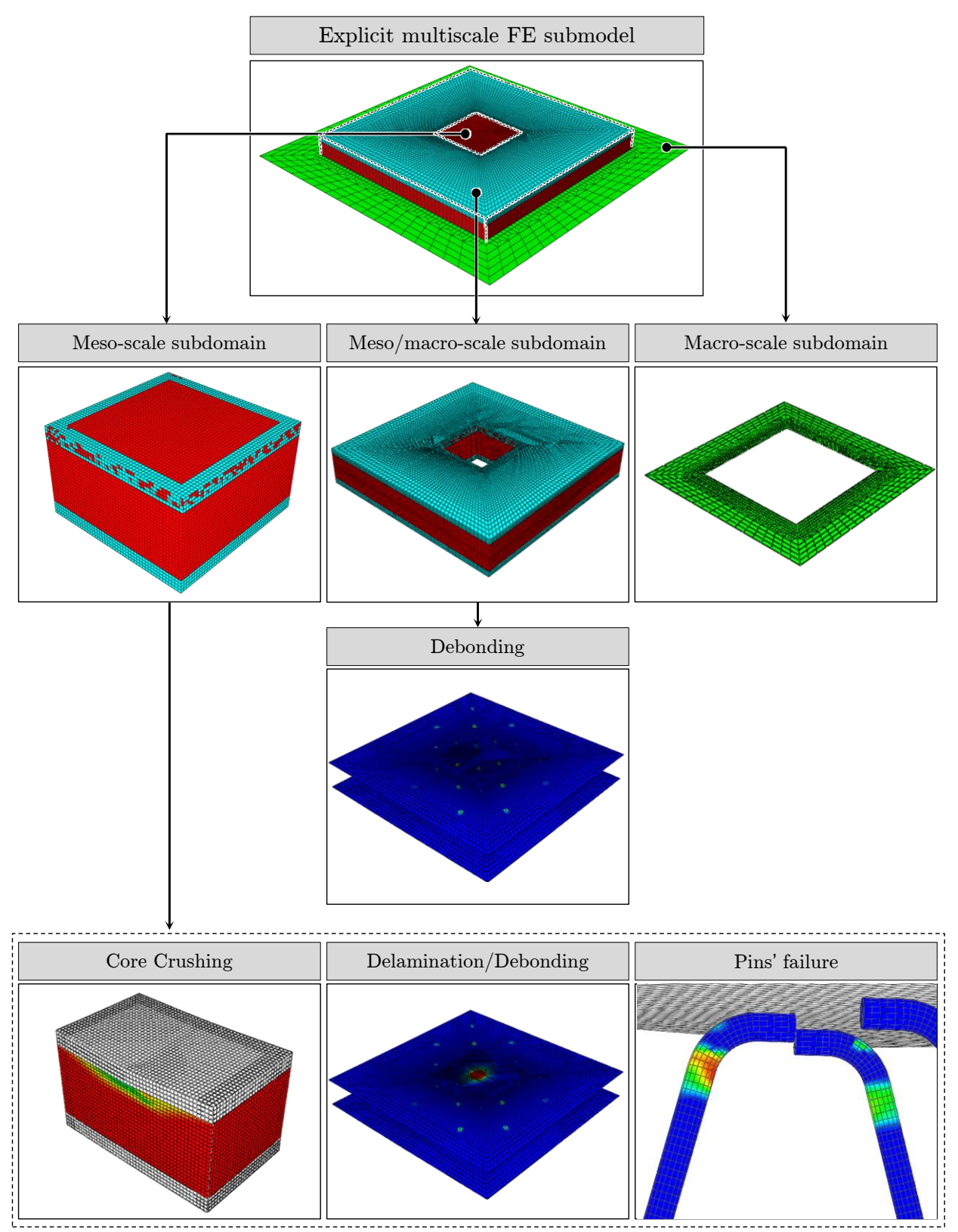

Fig. 4. Multiscale explicit FE submodel. Different areas of the structure can be modelled at different length-scales and their coupling be performed using the MST. The mechanical response of the structure can be correctly captured at all the length-scales of interest (both in terms of geometrical details and failure modes), while keeping the computational cost of the analysis to a minimum. 
(see Fig. 4). This can actually occur even at a significant distance from the impact location, as it might initiates as a result of the stress concentrations due to the pin reinforcements in the foam core layer.

\section{Macro-scale region}

Within this subdomain, the pin-reinforced sandwich structure is discretized using reduced-integration first-order quadrangular shell elements (S4R) with enhanced hourglass control. The equivalent homogenised $2 \mathrm{D}$ behaviour, i.e. the corresponding entire ABD matrix of the sandwich structure is computed exploiting the solid-to-shell homogenization described in Section 2. The discretization of the meso-scale subdomain (including the MST transition regions) required 1516 S4R elements.

\section{Discussion}

The key-aspects of the multiple length/time-scale framework for the virtual testing of large composite structures illustrated in this paper can be summarized as follows:

(i) coupling differently-discretized subdomains using the $\mathrm{MST}^{33}$ allows to minimize the areas of the structure modelled at the lowest (and therefore computationally costly) length-scales. Thus, the desired level of accuracy is attained only in selected portions of the model, which allows not to jeopardizing the computational cost of the analysis;

(ii) the response of different portions of the structure is simulated using different solvers, depending on where they are expected to provide the most efficient solution;

(iii) the exploitation of symmetries in the solid-to shell homogenization ${ }^{34}$ allows to strongly reduce the time required for the computation of equivalent homogenised properties;

(iv) the size of the resulting multiscale FE models can be minimized without any loss of accuracy at the lowest-scales; as a result, such FE models can be easily handled by high-performance computing resources, as well as modern laptop computers. As an example, the multiscale FE model of the helicopter rotor blade discussed in Section 3 consisted of a total number 1291202 DOFs (410846 nodes); simulations were performed using a laptop computer with an Intel i7-3610QM processor (2.3GHz Max Turbo Frequency), 8 CPUs and $8 \mathrm{~Gb}$ RAM for a total analysis time of approximately 6 hours.

\section{Conclusions}

In this paper, a multiple length/time-scale framework for the virtual testing of large composite structures is described. Its primitives and key-aspects are detailed through the analysis of a real-sized helicopter rotor blade subjected to a low-velocity impact.

The multiple length/time-scale framework enables a significant reduction of the 
CPU time required to compute the homogenized properties used in the higherscales of multiscale FE models; furthermore, it allows to minimize the areas of the structure that need to be modelled at the lowest length-scales, by opportunely coupling differently-discretized subdomains using a Mesh Superposition Technique.

The multiple length/time-scale framework investigated in this work represents a clear step towards the systematic and efficient virtual testing of large composite components.

\section{Acknowledgements}

The funding from Airbus Operations Ltd (UK) is gratefully acknowledged.

\section{Appendix A. Propagation of stress waves in non-uniform FE meshes using the Mesh Superposition Technique}

\section{A.1. Motivation}

The benefits provided by the MST in avoiding the artificial stress disturbances at the interfaces between differently-discretized subdomains are demonstrated in Ref. 33. In this example, we aim to investigate the capability of the MST to mitigate the spurious reflections of stress waves at the interfaces between differently-discretized subdomains.

\section{A.2. FE models}

An infinite bar of uniform cross-sectional area $\left(4 \times 4 \mathrm{~mm}^{2}\right)$ and made of an isotropic elastic material $\left(E=60 \mathrm{MPa}, \nu=0.25\right.$ and $\left.\rho=1600 \mathrm{~kg} / \mathrm{m}^{3}\right)$ was modelled in Abaqus. The initial $300 \mathrm{~mm}$ of the bar were discretized using conventional finite elements; the infinitely long idealization is achieved using infinite elements (CIN3D8).

For the region were conventional FE models were used, three different FE models of the infinite bar were created (see Fig. A.1):

\section{Fully Solid (FS) model}

the bar is discretized entirely using eight-noded reduced-integration solid elements (C3D8R) with enhanced hourglass control. Results obtained with this model are used as reference solution;

\section{Sudden Transition (ST) model}

the bar is discretized using eight-noded reduced-integration solid elements (C3D8R) with enhanced hourglass control for the initial $150 \mathrm{~mm}$; the remaining portion, eight-noded reduced-integration continuum shell elements (SC8R), with hourglass control and three integration points along the ele-

\section{MST model} ment's thickness, were used;

the sudden discretization-transition between solid (C3D8R) and continuum shell (SC8R) elements is replaced by a region where the MST is exploited. The length of the MST region is equal to $100 \mathrm{~mm}$. 


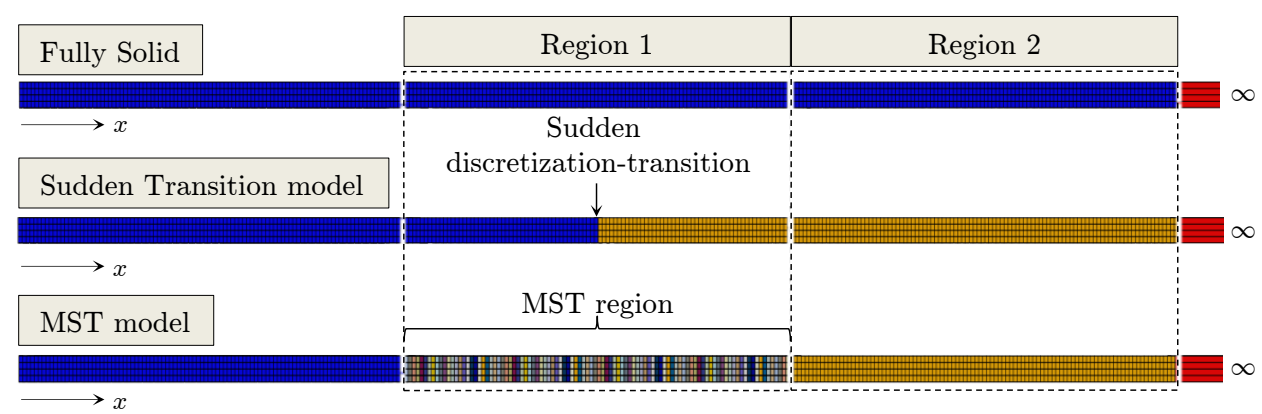

Fig. A.1. Three different FE models of the infinite bar: Fully Solid model, Sudden Transition model and MST model.

In Fig. A.1, Region 1 corresponds to the portion of the bar between $x=100 \mathrm{~mm}$ and $x=200 \mathrm{~mm}$, while Region 2 corresponds to the portion of the bar between $x=200 \mathrm{~mm}$ and $x=300 \mathrm{~mm}$. Region 1 includes the sudden distretization-transition for the ST model, and the entire MST region for the MST model.

\section{A.3. Stress-wave propagation analysis}

The applied displacement and boundary conditions considered are shown in Fig. A.2. The applied displacement consists of a finite discrete impulse defined

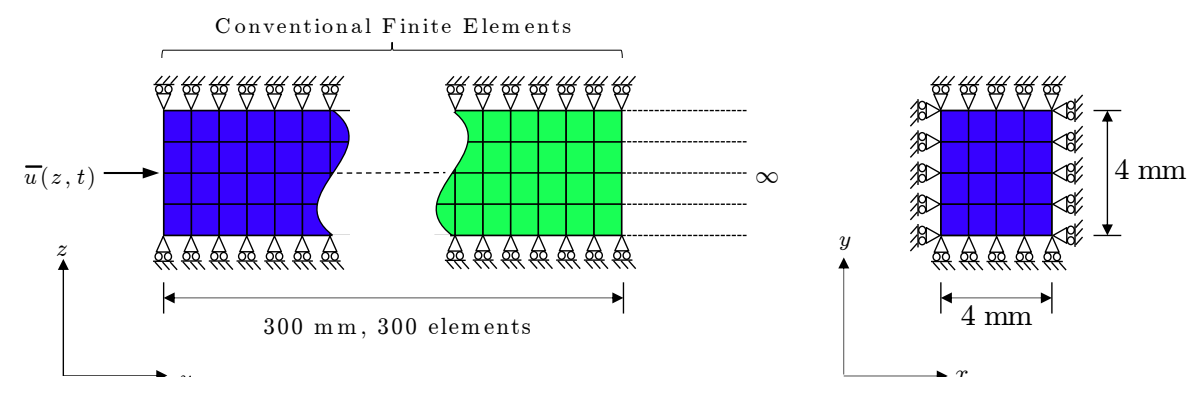

Fig. A.2. Applied displacement and boundary conditions for the stress-wave propagation study.

using Fourier series as

$$
\bar{u}(z, t)=\frac{\bar{u}(z)}{\kappa} \sum_{i=1}^{\kappa} \sin \left(2 \pi i f_{\min } t\right),
$$

where $\kappa=\frac{f_{\max }}{f_{\min }}$ is the number of terms in the Fourier series, $\bar{u}(z)$ is the amplitude of the applied displacement as a function of the out-of-plane coordinate, and $f_{\min }$ and $f_{\max }$ are, respectively, the minimum and maximum frequency of interest. 
In Fig. A.3, the evolution of the applied displacement as a function of time $(t)$ (Fig. A.3(a)) and of the $z$-coordinate (Fig. A.3(b)) is shown.

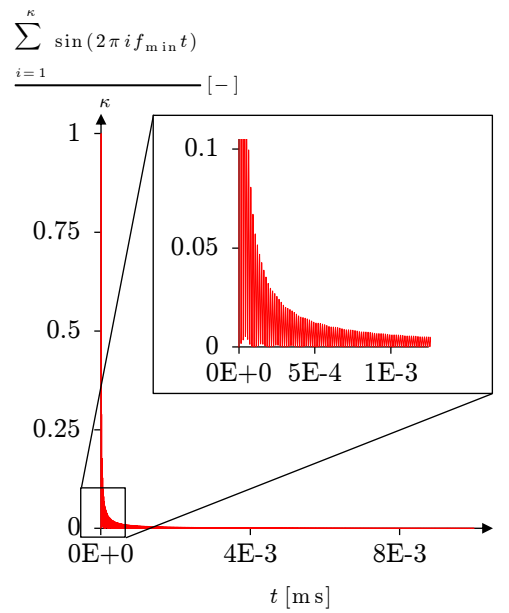

(a) Normalized amplitude of the applied displacement as a function of time.

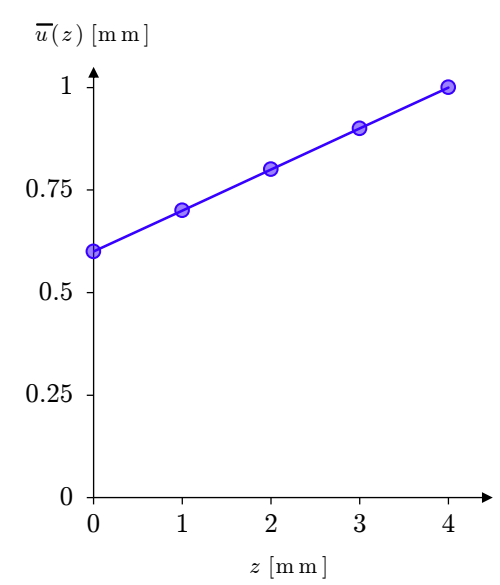

(b) Amplitude of the applied displacement as a function of the $z$-coordinate.

Fig. A.3. Time and space evolution of the applied displacement $\bar{u}(z, t)$. The resulting applied displaceent is a finite discrete impulse with variable amplitude along the through-the-thickness direction.

Simulations were performed using Abaqus/Standard. The analysis time-step was chosen so that the Courant-Friedrichs-Lewy condition ${ }^{51}$ (CFL) is fulfilled. The essence of the CFL condition is that, given a certain wave travelling across a discrete spatial grid, its amplitude can be correctly computed at fixed time steps of constant duration, only if the latter is smaller than the time needed for the wave to travel between two consecutive nodes of the spatial grid. In the simplified scenario of 1D wave propagation, the CFL condition can be expressed in terms of the Courant number $\mathcal{C}$ as

$$
\mathcal{C}=\frac{c \Delta t_{\max }}{\ell} \leq \mathcal{C}_{\max }
$$

where $c$ is the dilatational wave speed and $\ell$ is the characteristic length of the spatial grid. A value of $\mathcal{C}_{\max }=1$ is usually tolerated when implicit solvers are used. Nevertheless, a maximum time-step of $20 \mathrm{~ns}(\mathcal{C} \approx 0.122)$ was chosen for the analysis presented here.

\section{A.4. Results and discussion}

Fig. A.4 shows the internal energy $U$ of Region 1 and Region 2 (see Fig. A.1) as a function of time. Such internal energy corresponds to the energy carried by the 
stress waves travelling along the bar.

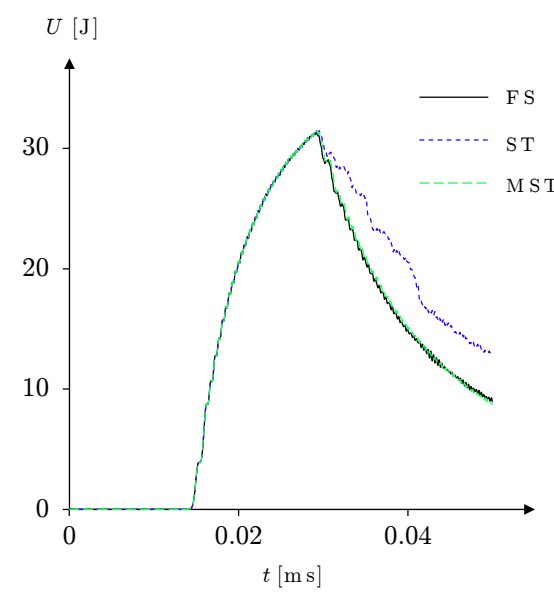

(a) Region 1.

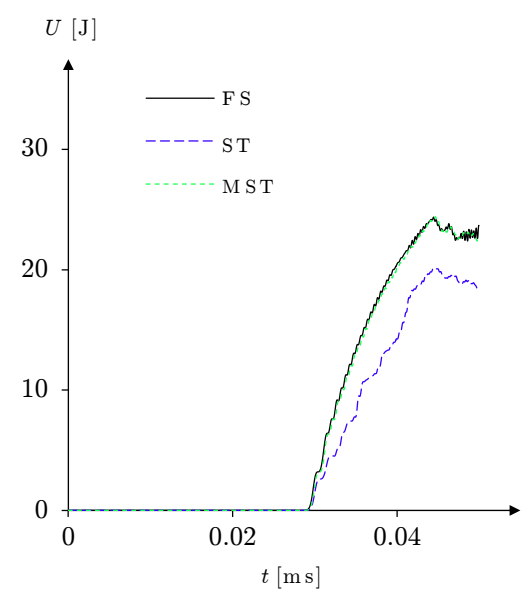

(b) Region 2 .

Fig. A.4. Time history of the internal energy of Region 1 (a) and Region 2 (b) associated with the propagation of the travelling stress waves along the infinite bar.

The stress waves enter into Region 1 at $t \approx 0.014 \mathrm{~ms}$ when the energy content of the latter starts increasing (see Fig. A.4(a)). At $t \approx 0.029 \mathrm{~ms}$, the stress waves travel from Region 1 into Region 2 (the energy content of Region 1 starts decreasing and, correspondingly, that of Region 2 starts increasing).

However, after $t \approx 0.029 \mathrm{~ms}$ the energy content of Region 1 computed with the ST model is higher than that computed with both the FS and MST model; a specular behaviour is associated to the energy content of Region 2. This evidence indicates that part of the stress waves travelling along the bar has been reflected by the sudden discretization-transition included in the ST model.

When compared to the reference solution obtained with the FS model, an energy loss of approximately $17 \%$ due to stress-wave reflections is observed when using the ST model. On the other hand, when using the MST for the progressive transition between the two differently-discretized subdomains, the energy loss associated to stress-wave reflections is almost completely eliminated (approximately $99 \%$ reduction if compared to the ST model).

The results presented in this section demonstrate that the $\mathrm{MST}^{33}$ allows to avoid, in addition to the artificial stress disturbances, also the spurious reflections of stress waves at the interfaces between differently-discretized subdomains. 


\section{References}

1. J. Pora, Composite Materials in the Airbus A380 - From History to Future - Proc. 13th Int. Conf. Compos. Mater., (2001).

2. M. Ostergaard, A.R. Ibbotson, O. Le Roux and A.M. Prior, Aeronaut. J., 1, 83-103, (2011).

3. J.F. Imbert, Airbus, Challenges in aircraft structure analysis - ESA/NAFEMS Seminar on Engineering Quality, Verification and Validation, Noordwijk, (2007).

4. A. Prior, Dassault Systémes, Nonlinear simulation of large scale aircraft structures implications for certification methodology and high-performance computing infrastructure - NAFEMS World Congress, (2009).

5. A. Prior, Dassault Systémes, Simulating damage and failure in aircraft structures $R A e S$ Conference: challenges for the next generation-concept to disposal, (2008).

6. T. Brown, Airbus, Working to meet the challenges of ext generation composite wing structural design - RAeS Conference: challenges for the next generation-concept to disposal, (2008).

7. European Aviation Safety Agency, Certification Specifications for Large Aeroplanes CS-25 Amendement 5, (2008).

8. R.W. McCune, C.G. Armstrong and D.J. Robinson, Int. J. Numer. Methods Eng., 49(6), 725-750, (2000).

9. C.G. Dávila, Comput. Syst. Eng., 5(2), 193-202, (1994).

10. D.M. Thompson, O.H. Griffin, Two-dimensional/three-dimensional global/local finite element analysis of composite laminates - Proc. 4th Ann American Soc. Composite Conf., 4th Tech. Conf., Technomic Publishing, Lancaster, PA, 234-243 (1989).

11. Z.P. Bažant, Comput. Methods Appl. Mech. Eng., 16(1), 91-100, (1978).

12. Z. Celep and D. Turhan, J. Sound Vib., 116(1), 15-23, (1987).

13. K. Shim, D. Monaghan and C. Armstrong, Eng. Comput., 18(3), 241-252, (2002).

14. N. Osawa, K. Hashimoto, J. Sawamura, T. Nakai and S. Suzuki, Mar. Struct., 20(3), 143-163, (2007).

15. Dassault Systémes Simulia Corp., ABAQUS 6.12 User's Manual, (2012).

16. J. Reinoso, A. Bázquez, A. Estefani, F. París, J. Canas, E. Arévalo and F. Cruz, Compos. Part B, 43(4), 1929-1942, (2012).

17. J. Reinoso, A. Bázquez, A. Estefani, F. París, J. Canas and E. Arévalo, Compos. Struct., 101, 274-289, (2013).

18. R.Krueger and T.K. O'Brien, Compos. Part A, 32(1), 25-44, (2001).

19. R.Krueger, J. Ratcliffe and P.J. Minguet, NASA/CR-200\%-214879 NIA Report No. 2007-0\%, (2007).

20. R. Krueger and P.J. Minguet, Compos. Struct., 81(1), 41-59, (2007).

21. R. Krueger, J.C. Ratcliffe and P.J. Minguet, Compos. Sci. Tech., 69(14) 2352-2362, (2009).

22. A. Gusev, J. Mech. Phys. Solids, 45(9), 1449-1459, (1997).

23. I. Gitman, H. Askes and L. Sluys, Eng. Fracture Mech., 74(16), 2518-2534, (2007).

24. C. Sun, R. Vaidya, Compos. Sci. Technol., 56(2), 171-179, (1996).

25. Z. Xia, Y. Zhang, F. Ellyin, Int. J. Solids Struct., 40(8), 1907-1921, (2003).

26. Z. Xia, C. Zhou, Q. Yong, X.Wang, Int. J. Solids Struct., 43(2) 266-278, (2006).

27. S. Li, Compos. Sci. Technol., 68(9), 1962-1974, (2008).

28. S. Li, Compos. Part A, 32(6), 815-826, (2001) .

29. S. Li and A.Wongsto, Mech. Mater., 36(7), 543-572, (2004).

30. J. Whitcomb, C. Chapman and X. Tang, J. Compos. Mater., 34(9), 724-747, (2000).

31. N.V. de Carvalho, S.T. Pinho and P. Robinson, Compos. Sci. Technol., 71(7), 969979, (2011). 
32. N.V. de Carvalho, S.T. Pinho and P. Robinson, arXiv:1012.3133v1, 1-18, (2010) .

33. L.Gigliotti and S.T. Pinho, Compos. Struct., 121, 395-405, (2015).

34. L.Gigliotti and S.T. Pinho, Compos. Struct., 132, 995-1005, (2015).

35. A. Gravouil and A. Combescure, Int. J. Numer. Methods Eng., 50(1), 199-225, (2001).

36. A. Combescure and A. Gravouil, Comput. Methods Appl. Mech. Eng., 191(11), 11291157, (2002).

37. Z.P. Bažant, Int. J. Multiscale Comput. Eng., 8(1), (2010)).

38. R.T. Whitcomb, Review of NASA supercritical airfoils -Proc. 9th Congr. Int. Council Aeron. Sci., Haifa, Israel, (1974).

39. G. Endres, Innovative sandwich constructions for aircraft application - Proc. 9th Int. Conf. Sandwich Struct., Pasadena, CA, (2010).

40. L. Gigliotti, S.T. Pinho, On the monotonic and cyclic compressive response of polymeric foam cores with TFC-reinforcements, In preparation for publication, (2016).

41. L. Gigliotti, S.T. Pinho, Prediction of the post-crushing compressive response of progressively crushable sandwich foam cores, Compos. Part A, Accepted for publication, (2016).

42. S.T. Pinho, L. Iannucci and P. Robinson, Compos. Part A, 37(5), 766-777, (2006).

43. HexFlow, RTM6-2 - Product Information. (2010).

44. T. Liu, Z. Deng and T. Lu, Int. J. Solids Struct., 45, 5127-5151, (2008).

45. S. Tabatabaei, S. Lomov and I. Verpoest, Compos. Struct., 107, 436-446, (2014).

46. G. Alfano and M.A. Crisfield, Int. J. Numer. Methods Eng., 50(7), 1701-1736, (2001).

47. Q. Yang and B. Cox, Int J Fract, 133(2), 107-137, (2005).

48. V.S. Deshpande and N.A. Fleck, J. Mech. Phys. Solids, 48(6-7), 1253-1283, (2000).

49. Z. Hashin, J. App. Mech., 47(2) 329-334, (1980).

50. R. Olsson and T.B. Block, Compos. Struct., 125, 81-87, (2015)

51. C. Richard, F. Kurt and L. Hans, IBM J. Res. Dev., 11(2), 215-234, (1967). 